\title{
Changes in miRNA expressions in the injured small intestine of mice following high-dose radiation exposure
}

\author{
MITSURU CHIBA $^{1}$, HARUKA UEHARA ${ }^{2}$, IKUMI NIIYAMA ${ }^{2}$, HARUKA KUWATA $^{2}$ and SATORU MONZEN $^{3}$ \\ ${ }^{1}$ Department of Bioscience and Laboratory Medicine, Graduate School of Health Sciences; \\ ${ }^{2}$ Department of Medical Technology, School of Health Sciences; ${ }^{3}$ Department of Radiation Science, \\ Graduate School of Health Sciences, Hirosaki University, Hirosaki, Aomori 036-8564, Japan
}

Received March 30, 2019; Accepted January 20, 2020

DOI: $10.3892 / \mathrm{mmr} .2020 .11054$

\begin{abstract}
The small intestine is one of the most highly regenerative and radiosensitive tissues in mammals, including humans. Exposure to high doses of ionizing radiation causes serious intestinal damage. Recently, several investigations have been conducted using radioprotective agents to determine ways for reducing intestinal damage caused by radiation exposure. However, a thorough understanding of functional changes occurring in the small intestine of mice exposed to high-dose radiation is necessary for developing novel and more potent radioprotective agents. In this study, we examined changes in microRNA (miRNA/miR) expressions in the small intestine of mice at $72 \mathrm{~h}$ after X-ray exposure (10 Gy). We identified seven upregulated miRNAs and six downregulated miRNAs in the small intestine of mice following radiation exposure using miRNA microarray analysis. Particularly, miR-34a-5p was highly expressed, which was confirmed by reverse transcription-quantitative PCR. Forkhead box P1 (Foxpl) was predicted to be a target of the mRNA of miR-34a-5p using OmicsNet. Decreased Foxpl expression in the small intestine following radiation exposure was confirmed, suggesting that Foxp1 expression recovery may induce the suppression of radiation-induced enteritis. Therefore, miR-34a-5p is a potential target molecule for developing novel radioprotective agents.
\end{abstract}

\section{Introduction}

The small intestine of mammals is one of the most sensitive organs to ionizing radiation (1). Leucine-rich repeat-containing $\mathrm{G}$ protein-coupled receptor 5(Lgr5)-positive stem cells present in the crypts of the small intestine are crucial for radiation-induced

Correspondence to: Dr Mitsuru Chiba, Department of Bioscience and Laboratory Medicine, Graduate School of Health Sciences, Hirosaki University, 66-1 Hon-cho, Hirosaki, Aomori 036-8564, Japan

E-mail: mchiba32@hirosaki-u.ac.jp

Key words: small intestine, radiation, gene expression, microRNA, microRNA-34a-5p intestinal regeneration (2). The death of Lgr5-positive stem cells due to high-dose radiation causes the disruption of intestinal homeostasis. The loss of the proliferative capacity of cells in the crypts of the small intestinal results in the depletion of many cells in the intestinal mucosa, leading to intestinal tract destruction. Exposure to radiation doses of $\geq 10$ Gy results in the development of classic gastrointestinal acute radiation syndrome (GI-ARS) in humans, usually within 5-10 days, if not appropriately treated (3). Other symptoms include the loss of appetite, diarrhea, infections, the loss of fluid and electrolytes, weight loss, malabsorption, and significant leukopenia $(4,5)$. In 1999, three individuals involved in the Tokai-mura JCO accident experienced severe acute radiation syndrome during; of these, two died owing to severe GI-ARS (6-8). The development of radioprotective agents that can mitigate the effects of high-dose radiation exposure is necessary and has been highlighted by many researchers. Verginadis et al, have demonstrated the radioprotective action of curcumin on the intestinal tract (9). Yamamoto et al, have reported that treatment with ascorbic acid prior to radiation exposure prevents fatal GI-ARS in mice (10). To develop effective radioprotective agents, thoroughly investigating the functional changes in the small intestine due to radiation exposure is essential.

MicroRNAs (miRNAs) are short single-stranded RNAs comprising approximately 20 bases. They are involved in regulating gene expression via the post-transcriptional regulation of messenger RNA (mRNA) and the inhibition of protein translation (11). Previously, we reported altered gene expression in cells exposed to radiation $(12,13)$. Other studies have also reported changes in mRNA expression in the small intestine following high-dose radiation exposure $(14,15)$. However, information on gene expression changes in miRNAs following such exposure is limited.

In this study, we analyzed the effects of changes in miRNA gene expression in the small intestine of mice following whole body irradiation with 10 Gy of X-rays.

\section{Materials and methods}

Mice andX-rayirradiation. All experiments were performed in accordance with The Guidelines for Animal Experimentation of the Hirosaki University. The procedures were approved and monitored by The Animal Research Committee of Hirosaki University (approval nos. G12003 and G19005). 
Male C57BL/6NJcl mice were purchased from CLEA, Japan. All mice were housed in a conventional animal room with 12-h light/dark cycles; all mice were provided food and water ad libitum. Eight-week-old mice were exposed to X-rays (MBR-1520R-3 X-ray machine, Hitachi Medical Corporation) at a rate of $1.0 \mathrm{~Gy} / \mathrm{min}(150 \mathrm{kVp}, 20 \mathrm{~mA}, 0.5 \mathrm{~mm}$ aluminum, and $0.3 \mathrm{~mm}$ copper filters). Mice were observed for up to 30 days after irradiation. Before dissection, mice were anesthetized with $2 \%$ isoflurane (Pfizer). To minimize distress as a humane end point, we performed cervical dislocation before death of the mice. Mice with severe diarrhea were sacrificed.

Fluorescent TdT-mediated dUTP nick end labeling (TUNEL) assay. The small intestine from mice at $72 \mathrm{~h}$ after non-irradiation and $10 \mathrm{~Gy}$ irradiation of X-ray was directly excised under anesthesia. For tissue analysis, the small intestine was fixed with 4\% paraformaldehyde solution in Dulbecco's phosphate-buffered saline (-) [D-PBS (-), pH 7.2]. The fixed small intestine was embedded in paraffin. Sections were cut at a thickness of $4 \mu \mathrm{m}$ and placed on glass slides. Paraffin-embedded sections of small intestine isolated from mice exposed to $10 \mathrm{~Gy}$ of X-rays on glass slides were deparaffinized with xylene and ethanol, followed by washing with D-PBS (-). Cell death analysis was performed using the DeadEnd ${ }^{\mathrm{TM}}$ Fluorometric TUNEL System (Promega) according to the manufacturer's instructions. Nuclei were stained using the ProLong Gold Antifade Reagent with 4',6-diamidino-2-phenylindole (Thermo Fisher Scientific, Inc.). The stained tissues were examined using a confocal laser scanning microscope LSM710 (Carl Zeiss).

Total RNA extraction. The non-irradiated and irradiated mice were sacrificed after $72 \mathrm{~h}$ for collection of small intestine. Total RNA was extracted from the small intestine using the Isogen II reagent (Nippon Gene) according to the manufacturer's instructions. RNA content in the small intestine was assessed using a NanoDrop spectrophotometer (NanoDrop Technologies). RNA samples had 260/280-nm absorbance ratios of 1.8-2.0. Total RNA quality was confirmed using the Agilent 2100 Bioanalyzer and Agilent RNA 6000 Pico kit (both Agilent Technologies, Inc.) as per the manufacturer's instructions.

Microarray analysis. Cyanine 3 (Cy3)-labeled miRNA was synthesized from total RNA obtained from the small intestines of irradiated (10 Gy, $n=4)$ and non-irradiated ( $0 \mathrm{~Gy}, \mathrm{n}=4)$ mice using the miRNA Complete Labeling Reagent and Hyb kit (Agilent Technologies). SurePrint G3 mouse miRNA microarray slides (8x60 K, v.21.0) were hybridized with the Cy3-labeled miRNA in a hybridization solution, which was prepared using a Gene Expression Hybridization kit (Agilent Technologies) as per the manufacturer's instructions. The images of $\mathrm{Cy} 3$ fluorescence signals on slides were obtained using the SureScan microarray scanner (Agilent Technologies) and were processed using the Feature Extraction software (v.10.7) based on the manufacturer's instructions. Expression data obtained were processed using the GeneSpring GX14.5 software (Agilent Technologies) to normalize all values to $90 \%$ percentile shift on the respective microarrays, followed by the normalization of the median expression level of all samples. miRNAs That were upregulated and downregulated by more than 2.000-fold in irradiated small intestine samples (10 Gy) compared with that in non-irradiated small intestine samples ( 0 Gy) were analyzed $(\mathrm{P}<0.05$, unpaired t-test). The Benjamini Hochberg FDR was used for multiple testing correction. Moreover, OmicsNet (https://www.omicsnet.ca/faces/home.xhtml) was used to predict target miRNA genes. To predict target genes of miRNAs and their pathways, TargetScan Mouse (http://www.targetscan.org/mmu_72/) analysis and WikiPathways (https://www.wikipathways. org/index.php/WikiPathways) analysis were performed using the GeneSpring 14.5 software (Agilent Technologies). Pathway data of Mus musculus were downloaded from WikiPathways.

Reverse transcription-quantitative PCR (RT-qPCR). Total RNA was used for synthesizing complementary DNAs (cDNAs) for miRNA expressions using the TaqMan ${ }^{\mathrm{TM}}$ miRNA RT kit and the prescribed 5xRT primer (both from Thermo Fisher Scientific, Inc.). Real-time PCR for miRNA expression was performed using a FastStart TaqMan probe master (Roche Diagnostics), 20x probe and the StepOne Plus Real-Time PCR system (Thermo Fisher Scientific, Inc.) under the following conditions: $10 \mathrm{~min}$ at $95^{\circ} \mathrm{C}$, followed by 45 cycles at $95^{\circ} \mathrm{C}$ for $15 \mathrm{sec}$ and $60^{\circ} \mathrm{C}$ for $60 \mathrm{sec}$. The comparative $\mathrm{Cq}$ method was used to assess miRNA expression levels. U6 small nuclear RNA was used as an internal control.

Western blotting. Western blotting was performed in the same protocol as before (16). In this study, the primary antibodies used were as follows: Anti-Forkhead box P1 (FoxP1) (D35D10) rabbit monocle antibody (no. 4402; Cell Signaling Technology, Inc.) and anti-Gapdh (D16H11) rabbit monocle antibody (no. 5174; Cell Signaling Technology, Inc.).

Statistical analysis. A Kaplan-Meier plot was used for assessing the survival of irradiated and non-irradiated mice. Statistical differences between irradiated and non-irradiated mice were determined using the log-rank test. $\mathrm{P}<0.05$ was considered to indicate a statistically significant difference. The Student's t-test was used to compare the results of the two groups.

\section{Results}

Exposure to 10 Gy X-rays in mice is fatal. We examined changes in body weight and survival time after exposure to 10 Gy X-rays. Weight loss was observed several days after irradiation (Fig. 1A). The 50\% survival time was approximately 9 days following radiation exposure (Fig. 1B), indicating that exposure to $10 \mathrm{~Gy} \mathrm{X}$-rays in mice is fatal. The TUNEL assay confirmed DNA damage in the intestinal epithelial cells following radiation exposure. At $72 \mathrm{~h}$ after radiation exposure, the green fluorescent TUNEL labeling increased, showing many positive images of the small intestinal pit site, particularly rich in small intestinal epithelial stem cells (Fig. 1C). These results demonstrate that cell death is induced in the small intestine of mice exposed to 10-Gy X-rays.

Identification of upregulated and downregulated miRNAs in the small intestine of mice following radiation exposure (10 Gy $X$-rays). The small intestines of 8 -week-old mice exposed to 10 Gy X-rays were extracted at $72 \mathrm{~h}$ after irradiation. Total RNA appeared to have an RNA integrity number of $>9.0$ (Fig. 2A). Change in miRNA expressions in the small intestine 
A

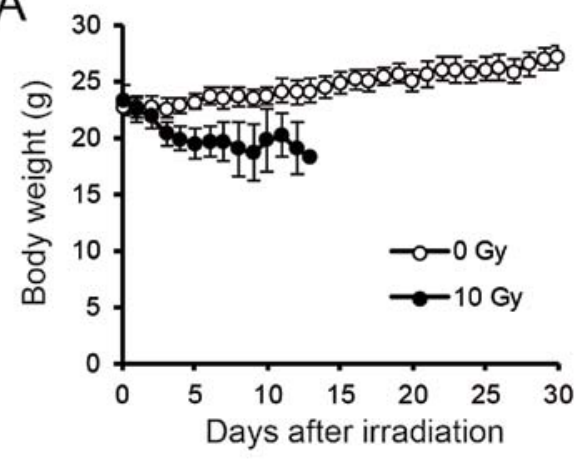

C

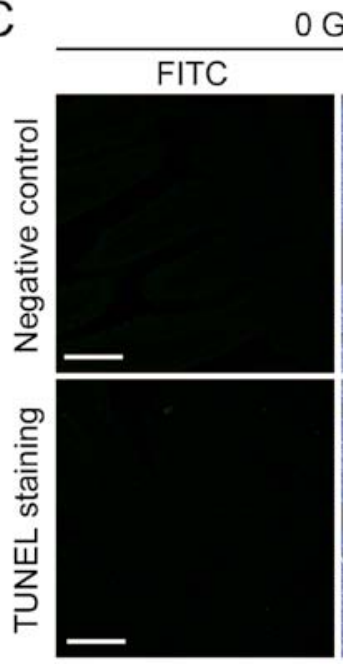

B
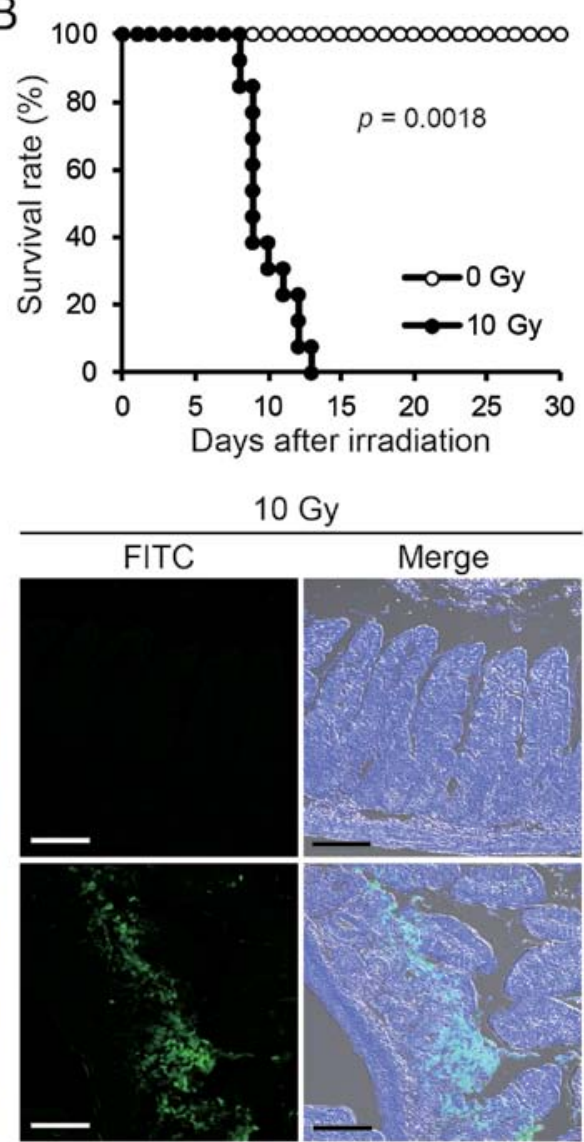

Figure 1. Effects of radiation exposure (10 Gy X-rays) on and injury in the small intestine. (A) Daily changes in body weight of irradiated C57BL/6 N male mice (10 Gy X-rays; $n=13)$ and non-irradiated mice (0 Gy; $n=5)$ up to 30 days after irradiation. The data are presented as the means \pm SD. (B) Kaplan-Meier plot for the survival of irradiated and non-irradiated mice. The statistical difference between irradiated and non-irradiated mice was determined using the log-rank test with $\mathrm{P}<0.05$ considered as statistically significant. (C) TUNEL assay of samples of small intestine following radiation exposure. Scale bar, $100 \mu \mathrm{m}$.

$$
\text { A }
$$

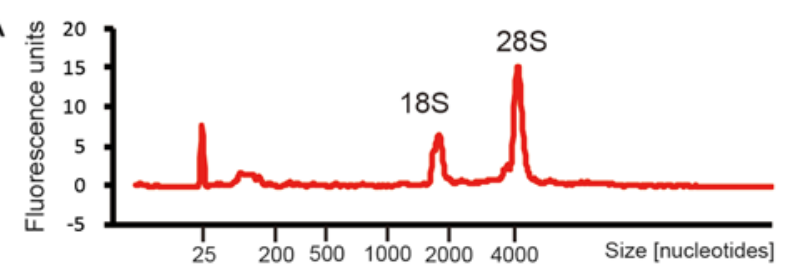

B

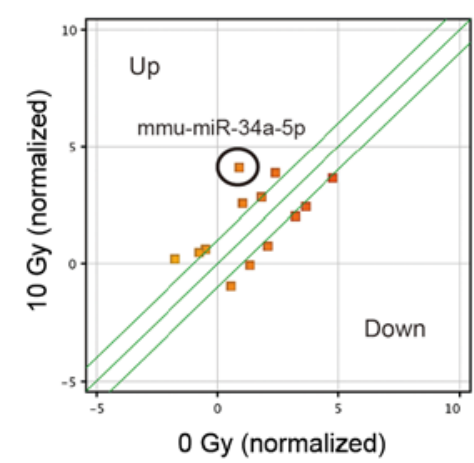

C

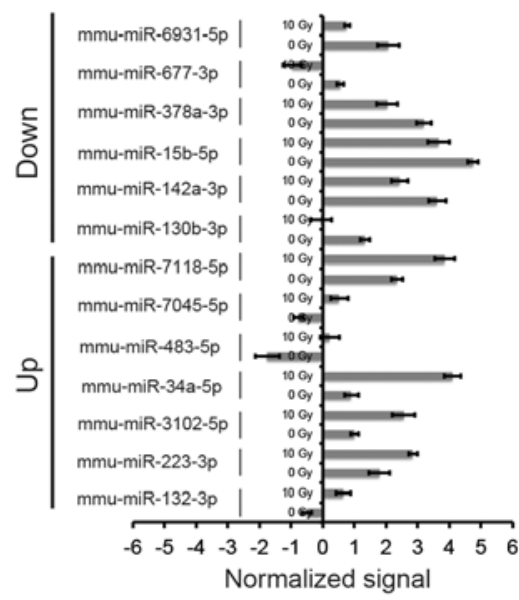

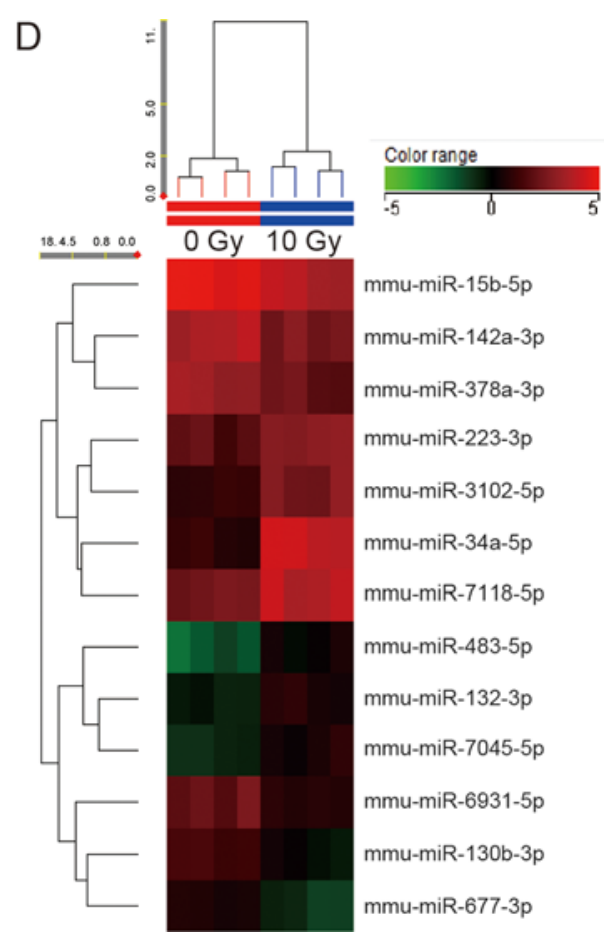

Figure 2. Changes in gene expressions in the small intestine of mice following radiation exposure (10 Gy X-rays). (A) Quality check of total RNA from small intestinal tissues using Agilent bioanalyzer. Gene expression of seven upregulated and six downregulated miRNAs in the small intestine of mice following radiation exposure using the SurePrint G3 mouse miRNA microarray. (B) Scatter plot analysis. (C) Variance of microarray data. Values are presented as the normalized signal mean $\pm \mathrm{SD}$. (D) Hierarchical clustering analysis. miRNA/miR, microRNA. 
Table I. Upregulated and downregulated miRNAs (>2.000-fold) in mouse small intestine at $72 \mathrm{~h}$ after exposure to $10 \mathrm{~Gy}$ of X-ray irradiation.

\begin{tabular}{llcr}
\hline Systematic_name & Mirbase accession no. & Fold change (10 Gy vs. 0 Gy) & P-value \\
\hline mmu-miR-132-3p & MIMAT0000144 & $2.209 \pm 0.131$ & 0.000332 \\
mmu-miR-223-3p & MIMAT0000665 & $2.081 \pm 0.536$ & 0.001907 \\
mmu-miR-3102-5p & MIMAT0014933 & $2.952 \pm 0.836$ & 0.000360 \\
mmu-miR-34a-5p & MIMAT0000542 & $9.137 \pm 0.624$ & 0.000004 \\
mmu-miR-483-5p & MIMAT0004782 & $3.911 \pm 1.901$ & 0.000393 \\
mmu-miR-7045-5p & MIMAT0027994 & $2.447 \pm 0.836$ & 0.000407 \\
mmu-miR-7118-5p & MIMAT0028133 & $2.826 \pm 0.642$ & 0.000346 \\
mmu-miR-130b-3p & MIMAT0000387 & $-2.626 \pm 0.314$ & 0.000525 \\
mmu-miR-142a-3p & MIMAT0000155 & $-2.261 \pm 0.771$ & 0.001618 \\
mmu-miR-15b-5p & MIMAT0000124 & $-2.117 \pm 0.331$ & 0.002784 \\
mmu-miR-378a-3p & MIMAT0003151 & $-2.241 \pm 0.283$ & 0.002295 \\
mmu-miR-677-3p & MIMAT0017246 & $-2.866 \pm 0.234$ & 0.000189 \\
mmu-miR-6931-5p & MIMAT0027762 & $-2.473 \pm 0.828$ & 0.000583 \\
\hline
\end{tabular}

miRNA, miR, microRNA; no, number.

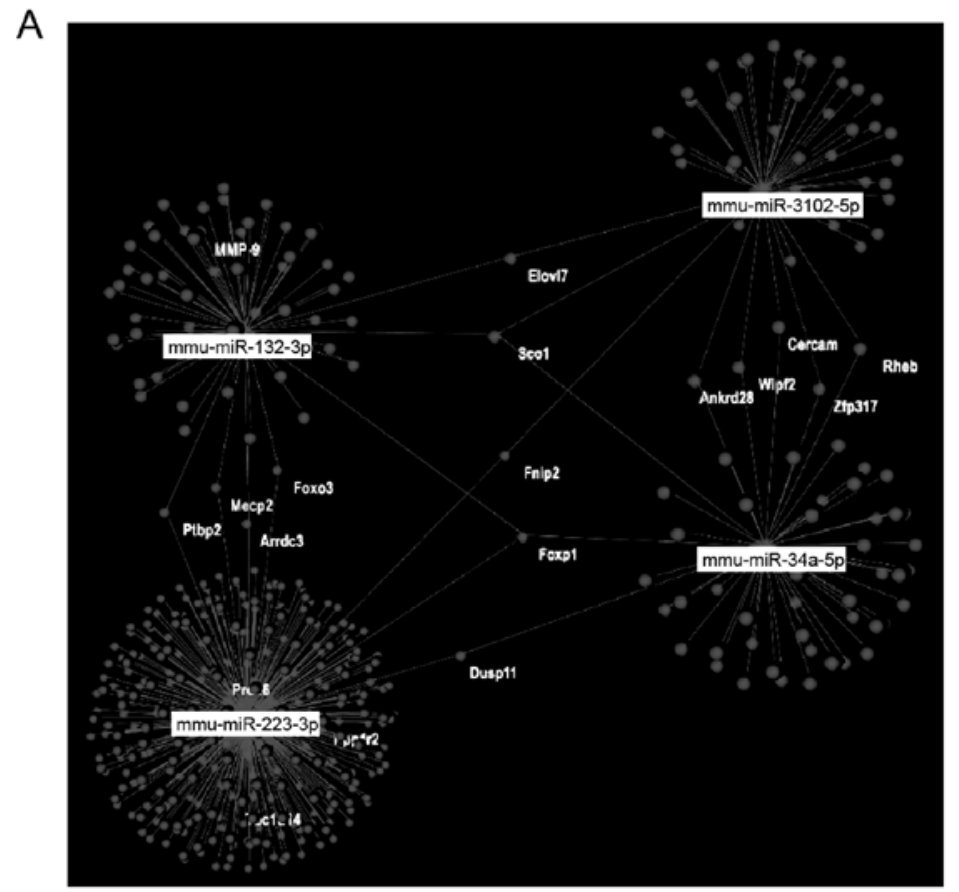

B

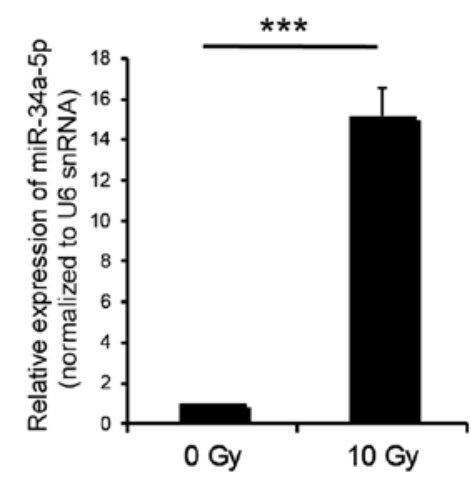

C

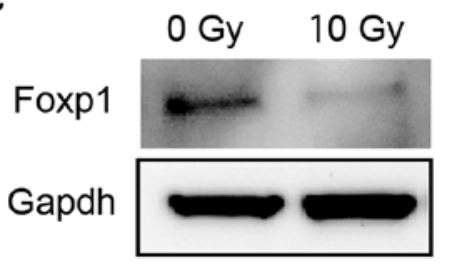

Figure 3. Search for target genes in upregulated and downregulated miRNAs in mouse small intestine following radiation exposure (10 Gy X-rays). (A) Network analysis of upregulated miRNAs and target genes using OmicsNet. (B) Gene expression changes of miR-34a-5p in small intestine of irradiated mice (10 Gy X-rays). Reverse transcription-quantitative PCR was performed using total RNA from mouse small intestine following radiation exposure. U6 small nuclear RNA was used as an internal control. ${ }^{* * *} \mathrm{P}<0.001$. (C) Expression changes of Foxp1 in small intestine of irradiated mice (10 Gy X-rays). Western blotting was performed using protein lysate from mouse small intestine following radiation exposure. GAPDH was used as the housekeeping gene. miRNA/miR, microRNA; snRNA, small nuclear RNA; Foxp1, Forkhead box P1.

were investigated using microarray analysis. We selected changes in miRNA expressions of over 2.000 -fold $(\mathrm{P}<0.05)$ and identified seven upregulated and six downregulated miRNA, respectively, (Fig. 2 and Table I).

Prediction of target genes in upregulated and downregulated miRNAs. To predict the target mRNA of the upregulated and downregulated miRNAs, we used OmicsNet. Foxp1 was predicted to be a target of the mRNAs of upregulated miR-34a-5p, miR-132-3p and miR-223-3p via analysis using OmicsNet (Fig. 3A). In particular, miR-34-5p was highly expressed, which was also validated using real-time PCR (Fig. 3B). Whereas, decreased Foxp1 expression in the small intestine following radiation exposure was confirmed using 
Table II. Top 10 pathways predicted to be regulated by miRNAs up- and downregulated $>2.000$-folds in the small intestine of mice exposed to $10 \mathrm{~Gy}$ of X-ray irradiation.

A, Upregulated miRNAs

\begin{tabular}{lr}
\hline Pathway name & P-value \\
\hline Mm_PluriNetWork_WP1763_89515 & $1.63 \times 10^{-18}$ \\
Mm_mRNA_processing_WP310_78419 & $7.06 \times 10^{-11}$ \\
Mm_Non-odorant_GPCRs_WP1396_69993 & $2.45 \times 10^{-8}$ \\
Mm_Metapathway_biotransformation_WP1251_94721 & $2.45 \times 10^{-8}$ \\
Mm_Myometrial_Relaxation_and_Contraction_Pathways_WP385_95806 & $2.45 \times 10^{-8}$ \\
Mm_IL-6_signaling_Pathway_WP387_72091 & $2.45 \times 10^{-8}$ \\
Mm_Focal_Adhesion-PI3K-Akt-mTOR-signaling_pathway_WP2841_94308 & $2.45 \times 10^{-8}$ \\
Mm_Focal_Adhesion_WP85_94410 & $2.45 \times 10^{-8}$ \\
Mm_Chemokine_signaling_pathway_WP2292_97515 & $2.45 \times 10^{-8}$ \\
Mm_Cytoplasmic_Ribosomal_Proteins_WP163_78425 & $8.49 \times 10^{-6}$ \\
\hline
\end{tabular}

B, Downregulated miRNAs

Pathway name

P-value

Mm_mRNA_processing_WP310_78419

$9.54 \times 10^{-43}$

Mm_PluriNetWork_WP1763_89515

$3.56 \times 10^{-33}$

Mm_Chemokine_signaling_pathway_WP2292_97515

$2.91 \times 10^{-31}$

Mm_MAPK_signaling_pathway_WP493_78412

$2.38 \times 10^{-29}$

Mm_TNF-alpha_NF-kB_Signaling_Pathway_WP246_69201

$1.94 \times 10^{-27}$

Mm_Insulin_Signaling_WP65_88446

$1.94 \times 10^{-27}$

Mm_Non-odorant_GPCRs_WP1396_69993

Mm_EGFR1_Signaling_Pathway_WP572_82883

$1.58 \times 10^{-25}$

Mm_Regulation_of_Actin_Cytoskeleton_WP523_71326

$1.58 \times 10^{-25}$

Mm_G_Protein_Signaling_Pathways_WP232_89955

$1.05 \times 10^{-21}$

$8.51 \times 10^{-20}$

miRNA, microRNA.

western blotting (Fig. 3C). These results suggest that Foxpl may be the target gene of miR-34a-5p and be involved in response to high-dose radiation exposure in the small intestine.

Prediction of pathways involved in target genes of miRNAs. To predict target genes of miRNAs and their pathways, WikiPathways analysis were performed. Using TargetScan, 201 genes were predicted as targets of the upregulated miRNAs (Table SI) and 857 of the downregulated miRNAs (Table SII). Under a p-value of $0.05,65$ and 122 pathways were predicted as target genes and pathways of the upregulated and downregulated miRNAs, respectively (Tables SIII and SIV). The top 10 pathways involved in up- and downregulated miRNAs are presented in Table II. Among these, the 'TNF- $\alpha \mathrm{NF}-\kappa \mathrm{B}$ Signaling Pathway' and 'Regulation of Actin Cytoskeleton' are presented in Fig. S1.

\section{Discussion}

This study revealed changes in the expression of various miRNA in the small intestine of mice following radiation exposure (10 Gy X-rays). In particular, miR-34a-5p was highly expressed at $72 \mathrm{~h}$ after radiation exposure, suggesting that this miRNA was the high-dose radio-responsive miRNA in the small intestine.

The body weight of irradiated mice decreased after several days of radiation exposure; in these mice, the $50 \%$ survival period was approximately 9 days (Fig. 1A and B). Fluorescent TUNEL assay confirmed that the small intestine was damaged by radiation exposure and TUNEL-positive cells were detected in the irradiation group and were not detected in the non-irradiated group. DNA damage was observed in the cells of the small intestine, particularly in stem cells, as the fluorescence of the small intestinal pit region strongly increased (Fig. 1C). Liu et al reported that intestinal Lgr5-positive stem cells underwent apoptosis following exposure to high-dose X-rays (17). Lgr5 is a marker of intestinal epithelial stem cells (18), and the death of Lgr5-positive cells indicates intestinal injury that is potentially lethal owing to poor regeneration ability of these cells. It will be interesting to examine if Lgr5-positive cells co-localize with TUNEL-positive cells in a future study.

We identified seven and six upregulated and downregulated miRNAs, respectively, in the small intestine of mice exposed to 10 Gy X-rays using miRNA microarray analysis. Particularly, 
miR-34a-5p overexpression by more than nine fold was observed in irradiated mice compared with that in non-irradiated mice. This suggests that miR-34a-5p is a radiation-responsive miRNA in the small intestine. Reportedly, miR-34a-5p regulates various target mRNAs involved in the cell cycle, cell proliferation, senescence, migration, and invasion, including cyclin-dependent kinase 4/6, E2F transcription factor 3, cyclin E2, hepatocyte growth factor receptor, B-cell lymphoma 2 (Bcl-2), NAD-dependent deacetylase sirtuin-1, Myc, Notch, and CD44 (19,20). Furthermore, miR-34a-5p primarily induces p53-mediated apoptosis and cell cycle arrest in the G1 phase and during senescence $(21,22)$. Radiation exposure has been reported to increase miR-34a-5p expression in different types of cells such as breast cancer cells (23), lung cancer cells (24), prostate cancer cells (25), lymphocytes (26), spleen cells (21), and colorectal cancer cells (27). However, the present study is the first to report on the increased miR-34a-5p expression in the small intestine following high-dose radiation exposure.

Reportedly, elevated miR-34a-5p expression in the small intestine suppresses Bcl-2 and Notch expressions, thus promoting apoptosis and resulting in the deterioration of the small intestine. Conversely, it is expected that increased miR-34a-5p expression suppresses RAD51, reduces double-strand break repair, and enhances radiosensitivity. Interestingly, miR-34a-5p has been reported to an indicator of radiation exposure (28); this report revealed that the serum miR-34a-5p level increases following radiotherapy in patients with breast cancer. In the future, it will necessary to examine the possibility of using serum miR-34a-5p as a biomarker for determining intestinal high-dose radiation exposure.

Foxpl belongs to subfamily $\mathrm{P}$ of the forkhead box transcription factor family and plays important roles in organ development (29). Moreover, it is a valuable prognostic biomarker in lymphoma (30). However, the role of Foxpl in the intestinal tissue is slightly unclear. De Smedt et al, suggested that the loss of FOXP1 was associated with decreased survival of patients with colorectal cancer (CRC) and affected CRC proliferation and inflammatory responses (31). Presumably, Foxpl is a target of miR-34a-5p; however, the relationship between high-dose radiation and Foxpl expression has not been reported till date. The maintenance of Foxpl expression with the use of an miR-34a-5p inhibitor may suppress radiation-induced enteritis; thus, an miR-34a-5p inhibitor may be used as a novel radioprotective agent.

Gene expression is predicted to be upregulated and downregulated by radiation exposure, and pathway analysis showed the enhancement of 'TNF- $\alpha$ NF- $\kappa$ B Signaling Pathway' (Table II). TNF- $\alpha$ stimulation and ROS generation following radiation cause $\mathrm{NF}-\kappa \mathrm{B}$ activation via I $\kappa \mathrm{B}$ kinase signaling and induce enteritis $(32,33)$. The predicted enhancement of the 'TNF- $\alpha$ NF- $\kappa$ B Signaling Pathway' suggests radiation-induced enteritis (Table II). Recently, radioprotective agents, such as $\alpha$-lipoic acid and L-carnitine, have been studied, which alleviate radiation-induced enteritis as a side effect during radiotherapy $(34,35)$. Understanding the underlying molecular mechanisms may lead to the development of novel effective radioprotective agents.

In this study, we examined miRNA expression in the small intestine after $72 \mathrm{~h}$ of radiation exposure using 8 week-old mice. In a previous study, we examined miRNA expression at various time-course with a high-dose ( $7 \mathrm{~Gy}$ ) of irradiation and observed a significant difference at $72 \mathrm{~h}$ compared with that at baseline (36). Based on these findings, changes after $72 \mathrm{~h}$ were examined in this study; however, there may be a possibility that the results of these two studies differ owing to the use of two radiation doses. In the future, analyzing miR-34a-5p expression at various time-course with 10 Gy irradiation will be necessary. In addition, the effects of radiation on the small intestine may vary depending on the age of the mouse. However, since it is difficult to use mice of all ages, 8 weeks of age was used as a representative of mice that reached adulthood. We plan to investigate the effects on the intestinal tract of children and older mice in the future.

\section{Acknowledgements}

Not applicable.

\section{Funding}

The present study was supported in part by The JSPS KAKENHI (grant nos. JP25670264, JP17H04761 and JP17K19779) and a grant from The Takeda Science Foundation in 2016.

\section{Availability of data and materials}

The datasets used and/or analyzed during the present study are available from the corresponding author upon reasonable request.

\section{Authors' contributions}

$\mathrm{MC}$ was a major contributor in performing the experiments and writing the manuscript. HU, IN, HK and SM helped conduct the experiments. All authors read and approved the final manuscript.

\section{Ethics approval and consent to participate}

All experiments were performed in accordance with The Guidelines for Animal Experimentation of the Hirosaki University. The procedures were approved and monitored by The Animal Research Committee of Hirosaki University (approval nos. G12003 and G19005).

\section{Patient consent for publication}

Not applicable.

\section{Competing interests}

The authors declare that they have no competing interests.

\section{References}

1. International Atomic Energy Agency: Diagnosis and treatment of radiation injures. IAEA Safety Report Series 2. IAEA, Vienna, 1998.

2. Metcalfe C, Kljavin NM, Ybarra R and de Sauvage FJ: Lgr5+ stem cells are indispensable for radiation-induced intestinal regeneration. Cell Stem Cell 14: 149-159, 2014.

3. Leibowitz BJ, Wei L, Zhang L, Ping X, Epperly M, Greenberger J, Cheng $\mathrm{T}$ and $\mathrm{Yu} \mathrm{J}$ : Ionizing irradiation induces acute haematopoietic syndrome and gastrointestinal syndrome independently in mice. Nat Commun 5: 3494, 2014. 
4. Berger ME, Christensen DM, Lowry PC, Jones OW and Wiley AL: Medical management of radiation injuries: Current approaches. Occup Med (Lond) 56: 162-172, 2006.

5. Macià I Garau M, Lucas Calduch A and López EC: Radiobiology of the acute radiation syndrome. Rep Pract Oncol Radiother 16 : 123-130, 2011.

6. Tanaka SI: Summary of the JCO criticality accident in Tokai-mura and a dose assessment. J Radiat Res 42 (Suppl): S1-S9, 2001.

7. Sasaki MS, Hayata I, Kamada N, Kodama Y and Kodama S Chromosome aberration analysis in persons exposed to low-level radiation from the JCO criticality accident in Tokai-mura. J Radiat Res 42: S107-S116, 2001.

8. Asano S: Current status of hematopoietic stem cell transplantation for acute radiation syndromes. Int J Hematol 95: 227-231, 2012.

9. Verginadis II, Kanade R, Bell B, Koduri S, Ben-Josef E and Koumenis C: A novel mouse model to study image-guided radiation-induced intestinal injury and preclinical screening of radioprotectors. Cancer Res 77: 908-917, 2017.

10. Yamamoto T, Kinoshita M, Shinomiya N, Hiroi S, Sugasawa H, Matsushita Y, Majima T, Saitoh D and Seki S: Pretreatment with ascorbic acid prevents lethal gastrointestinal syndrome in mice receiving a massive amount of radiation. J Radiat Res 51 145-156, 2010.

11. Mohr AM and Mott JL: Overview of microRNA biology. Semin Liver Dis 35: 3-11, 2015.

12. Chiba M, Miura T, Kasai K, Monzen S, Kashiwakura I, Yasue $\mathrm{H}$ and Nakamura T: Identification of up-regulated and down-regulated cis-natural antisense transcripts in the human B lymphoblastic cell line IM-9 after X-ray irradiation. Mol Med Rep 5: 1151-1157, 2012.

13. Chiba M: Radiation-responsive transcriptome analysis in human lymphoid cells. Radiat Prot Dosimetry 152: 164-167, 2012.

14. Strup-Perrot C, Mathé D, Linard C, Violot D, Milliat F, François A, Bourhis J and Vozenin-Brotons MC: Global gene expression profiles reveal an increase in mRNA levels of collagens, MMPs, and TIMPs in late radiation enteritis. Am J Physiol Gastrointest Liver Physiol 287: G875-G885, 2004.

15. Zheng J, Wang J, Pouliot M, Authier S, Zhou D, Loose DS and Hauer-Jensen M: Gene expression profiling in non-human primate jejunum, ileum and colon after total-body irradiation: A comparative study of segment-specific molecular and cellular responses. BMC Genomics 16: 984, 2015.

16. Chiba M, Kubota S, Sakai A and Monzen S: Cell-to-cell communication via extracellular vesicles among human pancreatic cancer cells derived from the same patient. Mol Med Rep 18: 3989-3996, 2018

17. Liu Z, Liu H, Jiang J, Tan S, Yang Y, Zhan Y and Wu B: PDGF-BB and bFGF ameliorate radiation-induced intestina progenitor/stem cell apoptosis via Akt/p53 signaling in mice. Am J Physiol Gastrointest Liver Physiol 307: G1033-G1043, 2014.

18. Barker N, van Es JH, Kuipers J, Kujala P, van den Born M, Cozijnsen M, Haegebarth A, Korving J, Begthel H, Peters PJ and Clevers $\mathrm{H}$ : Identification of stem cells in small intestine and colon by marker gene Lgr5. Nature 449: 1003-1007, 2007.

19. Hermeking H: The miR-34 family in cancer and apoptosis. Cell Death Differ 17: 193-199, 2010.

20. Misso G, Di Martino MT, De Rosa G, Farooqi AA, Lombardi A, Campani V, Zarone MR, Gullà $\mathrm{A}$, Tagliaferri $\mathrm{P}$, Tassone $\mathrm{P}$ and Caraglia M: Mir-34: A new weapon against cancer? Mol Ther Nucleic Acids 3: e194, 2014.

21. He L, He X, Lim LP, de Stanchina E, Xuan Z, Liang Y, Xue W, Zender L, Magnus J, Ridzon D, et al: A microRNA component of the p53 tumour suppressor network. Nature 447: 1130-1134, 2007.

22. Tarasov V, Jung P, Verdoodt B, Lodygin D, Epanchintsev A, Menssen A, Meister G and Hermeking H: Differential regulation of microRNAs by p53 revealed by massively parallel sequencing. miR-34a is a p53 target that induces apoptosis and G1-arrest. Cell Cycle 6: 1586-1593, 2007.
23. Stankevicins L, Almeida da Silva AP, Ventura Dos Passos F, Dos Santos Ferreira E, Menks Ribeiro MC, G David M, J Pires E, Ferreira-Machado SC, Vassetzky Y, de Almeida CE and de Moura Gallo CV: MiR-34a is up-regulated in response to low dose, low energy X-ray induced DNA damage in breast cells. Radiat Oncol 8: 231, 2013.

24. Salzman DW, Nakamura K, Nallur S, Dookwah MT, Metheetrairut C, Slack FJ and Weidhaas JB: miR-34 activity is modulated through 5'-end phosphorylation in response to DNA damage. Nat Commun 7: 10954, 2016.

25. John-Aryankalayil M, Palayoor ST, Makinde AY, Cerna D, Simone CB II, Falduto MT, Magnuson SR and Coleman CN: Fractionated radiation alters oncomir and tumor suppressor miRNAs in human prostate cancer cells. Radiat Res 178: 105-117, 2012.

26. Girardi C, De Pittà C, Casara S, Sales G, Lanfranchi G, Celotti L and Mognato M: Analysis of miRNA and mRNA expression profiles highlights alterations in ionizing radiation response of human lymphocytes under modeled microgravity. PLoS One 7: e31293, 2012

27. Liu C, Zhou C, Gao F, Cai S, Zhang C, Zhao L, Zhao F, Cao F, Lin J, Yang Y, et al: MiR-34a in age and tissue related radio-sensitivity and serum miR-34a as a novel indicator of radiation injury. Int J Biol Sci 7: 221-233, 2011.

28. Halimi M, Shahabi A, Moslemi D, Parsian H, Shari SM, Satire R, Yeganeh $F$ and Zabihi E: Human serum miR-34a as an indicator of exposure to ionizing radiation. Radiat Environ Biophys 55: 423-429, 2016.

29. Usui N, Araujo DJ, Kulkarni A, Co M, Ellegood J, Harper M, Toriumi K, Lerch JP and Konopka G: Foxp1 regulation of neonatal vocalizations via cortical development. Genes Dev 31: 2039-2055, 2017

30. Gascoyne DM and Banham AH: The significance of FOXP1 in diffuse large B-cell lymphoma. Leuk Lymphoma 58: 1037-1051, 2017.

31. De Smedt L, Palmans S, Govaere O, Moisse M, Boeckx B, De Hertogh G, Prenen H, Van Cutsem E, Tejpar S, Tousseyn T and Sagaert X: Expression of FOXP1 and colorectal cancer prognosis. Lab Med 46: 299-311, 2015.

32. He LX, Wang JB, Sun B, Zhao J, Li L, Xu T, Li H, Sun JQ, Ren J, Liu R, et al: Suppression of TNF- $\alpha$ and free radicals reduces systematic inflammatory and metabolic disorders: Radioprotective effects of ginseng oligopeptides on intestinal barrier function and antioxidant defense. J Nutr Biochem 40: 53-61, 2017.

33. Kalita B, Ranjan R, Singh A, Yashavarddhan MH, Bajaj S and Gupta ML: A combination of podophyllotoxin and rutin attenuates radiation induced gastrointestinal injury by negatively regulating NF- $\kappa \mathrm{B} / \mathrm{p} 53$ signaling in lethally irradiated mice. PLoS One 11: e0168525, 2016

34. Tas S, Ozkan OF, Cikman O, Kiraz A, Akgun Y and Karaayvaz M: L-carnitine has a protective effect on the colonic mucosa during abdominopelvic radiotherapy in rats. Acta Cir Bras 31: 615-620, 2016.

35. Jeong BK, Song JH, Jeong H, Choi HS, Jung JH, Hahm JR, Woo SH, Jung MH, Choi BH, Kim JH and Kang KM: Effect of alpha-lipoic acid on radiation-induced small intestine injury in mice. Oncotarget 7: 15105-15117, 2016.

36. Chiba M, Monzen S, Iwaya $\mathrm{C}$, Kashiwagi $\mathrm{Y}$, Yamada $\mathrm{S}$, Hosokawa Y, Mariya Y, Nakamura T and Wojcik A: Serum miR-375-3p increase in mice exposed to a high dose of ionizing radiation. Sci Rep 8: 1302, 2018.

This work is licensed under a Creative Commons Attribution-NonCommercial-NoDerivatives 4.0 International (CC BY-NC-ND 4.0) License. 